\title{
Subungual Invasive Squamous Cell Carcinoma with Doubtful Underlying Bone Invasion: A Treatment Proposal
}

\author{
Judith Dominguez-Cherit \\ Department of Dermatology, Instituto Nacional de Ciencias Médicas y Nutrición "Salvador Zubiràn", \\ Mexico City, Mexico
}

\section{Established Facts}

- Fact 1. In subungual squamous cell carcinoma, a nail biopsy is necessary to demonstrate it; if an invasion is documented, it is necessary to rule out bone invasion.

- Fact 2. With a single biopsy, it is very difficult to define the depth of invasion so it is necessary to remove the whole nail apparatus or the whole lesion and look for bone invasion with an MRI.

- Fact 3. If bone invasion is documented by MRI, sometimes it is difficult to differentiate from edema, but in case of doubt, digit amputation has to be performed.

\section{Novel Insights}

- Squamous cell carcinoma can invade or not phalanx, an ungual biopsy cannot assure invasion.

- To rule out invasive squamous cell carcinoma, it is necessary to remove the whole lesion, and to rule out bone invasion, an MRI is performed.

- After nail biopsy, bone invasion and medullar inflammations are difficult to distinguish.

- Bone biopsy during excision of nail apparatus is necessary before deciding phalanx amputation.

\section{Keywords}

Subungual squamous cell carcinoma · Bone invasion ·

Amputation

\section{Abstract \\ Introduction: Subungual squamous cell carcinoma (sSCC) is the most frequent malignant tumor of the nail unit. When suspecting sSCC, accurate diagnosis is supported by nail bi- opsy. Depth of invasion and histopathologic signs of human papillomavirus (HPV) infection are related to an unfavorable}

(C) 2020 S. Karger AG, Basel

www.karger.com/sad

Karger ${ }^{\prime}=$ prognosis. In this case, it is necessary to rule out bone invasion by radiography or MRI, and in case of doubt, amputation is indicated. Case Presentation: We present 2 cases of sSCC with a confirmatory biopsy of sSCC with HPV and invasion; in both cases, MRI was difficult to distinguish between bone invasion and bone marrow inflammation. Before amputating the distal phalanx, we decided to perform a bone biopsy during the excisional surgery. Neither case required amputation. Conclusion: We propose that when an invasive sSCC is present and an MRI is doubtful of bone invasion, before amputating, a bone biopsy should be performed.

(c) 2020 S. Karger AG, Base

Judith Dominguez-Cherit

Department of Dermatology

Instituto Nacional de Ciencias Médicas y Nutrición "Salvador Zubiràn"

Vasco de Quiroga No. 15, Mexico City 14080 (Mexico)

dominguez.judith@gmail.com 


\section{Introduction}

Subungual squamous cell carcinoma (sSCC) is the most frequent malignant tumor of the nail unit, predominantly affecting people in the fifth decade of life [1] and is also associated with high-risk human papillomavirus (HPV) 16 infection in $80 \%$ of the cases. When suspecting sSCC, accurate diagnosis is supported by nail biopsy, and after its confirmation, removal of the whole nail unit is mandatory. However, when an invasive sSCC is diagnosed, depth of invasion and histopathologic signs of HPV infection are related to an unfavorable prognosis. In this case, radiography is mandatory to rule out bone invasion, and in case of doubt, a magnetic resonance should be performed. In some cases, the distinction between tumor infiltration and other causes of bone marrow edema in the underlying bone is difficult to assess. The treatment of choice in case of bone invasion is amputation of the involved digit [2].

\section{Case Report/Case Presentation}

We report 2 cases: a 33-year-old male, with an 8-month rapid destructive nail tumor of the right thumb (shown in Fig. 1), and a 53-year-old male, with a rapidly growing verrucous and eczematous plaque on the lateral nail fold of the right third finger. Both of them had a diagnostic biopsy of invasive nail unit sSCC (invasive sSCC), associated with high-risk HPV infection, but because it was an incisional biopsy, it was difficult to confirm the exact depth of infiltration, so an MRI was performed in search of bone invasion. In radiological findings of both cases, it was difficult to differentiate between bone invasion and secondary edema due to the previous biopsy (shown in Fig. 2) After discussing with both patients the uncertainty of tumor infiltration in the underlying bone and the intention of preventing digit amputation, an alternative procedure was proposed. First, removal of the whole nail unit was carried out, and during this surgical process, a bone biopsy to be performed with a hand gouge by just cutting the distal portion of the distal phalanx at the exact site of the overlying tumor. The surgical defect will be closed with a skin graft, while waiting until the final bone histopathology report was rendered. If osseous malignant invasion was confirmed, amputation surgery of the phalanx will be indicated. However, if the bone was negative for tumor infiltration
Fig. 1. The proximal destruction of the nail plate and partial onychomadesis, also the tumor emerging from the underlying proximal nail fold.

Fig. 2. In different sagittal cuts, the opacity (arrow) more accentuated in the distal phalanx of the 3 rd cut, difficult to differentiate between bone invasion and medullar edema.

Subungual Squamous Cell Carcinoma and Bone Invasion
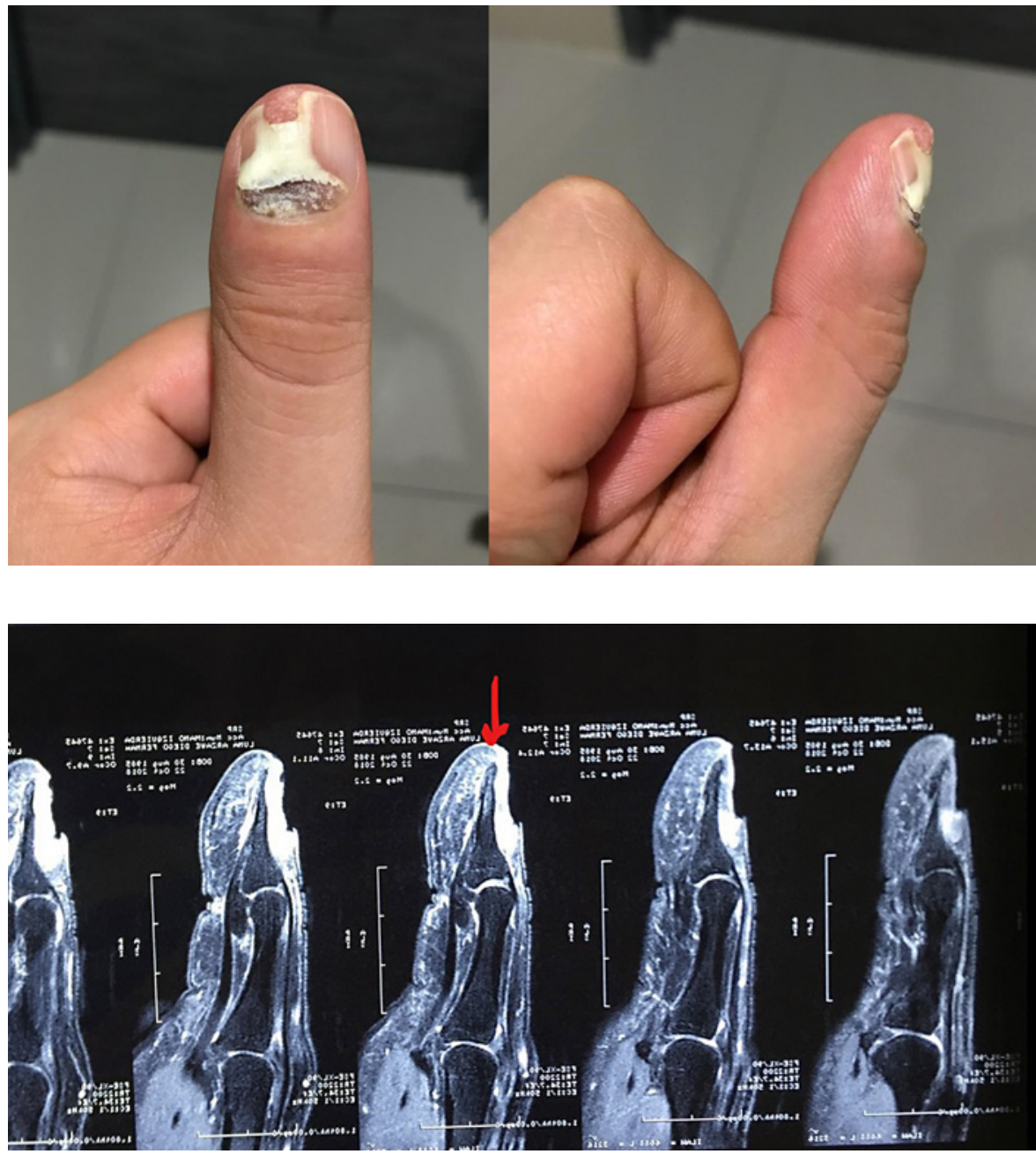

Skin Appendage Disord 2021;7:108-111 DOI: $10.1159 / 000511740$ 
the digit could be preserved. Both patients agreed to the nail unit block excision and bone biopsy; in both cases, there was no histologic evidence of bone invasion and the phalanges of the digits were preserved. After 18 months of follow-up, both of them are free of disease.

\section{Discussion}

sSCC is the most frequent malignant tumor of the nail unit. It presents predominantly on the thumb and index finger of people in the fifth decade of life. Risk factors for sSCC include trauma, immunosuppression, chronic paronychia, oral exposure to arsenic or pesticides, ionizing radiation exposure, dyskeratosis congenita, and HPV infection with subtypes $2,11,18,26,31,34,35,52,56,58$, 73 , and especially type 16 (in $60-80 \%$ of cases) $[1,3]$. sSCC has different clinical presentations with no specific or pathognomonic features, the reason why, it is frequently misdiagnosed, leading to an average delay of $>4$ years before receiving appropriate treatment. Infiltration into the onychodermis depth of invasion and association with HPV infection are related to a bad prognosis. Bony invasion is observed in approximately $20 \%$ of invasive sSCC cases $[2,3]$. Because of the rarity of this nail unit tumor, there are not enough published data regarding the treatment for this subtype of squamous cell carcinoma at the subungual location. There are proposed guidelines neither in the chapter of Cutaneous Squamous Cell Carcinoma of the 2020 version of the National Comprehensive Cancer Network (NCCN 2020) nor in the AJJC-8 or the $\mathrm{BWH}[4,5]$. It is well known, from different case report series, that amputation is the treatment of choice for invasive sSCC with bone involvement. The first approach to a suspicious case is to perform an appropriate nail biopsy. If an invasive sSCC is histologically confirmed, it is necessary to perform a radiograph or computed tomography to evaluate bone involvement, a feature that, in some cases, could be difficult to interpret $[2,6]$ This is the reason why the suggested best imaging study to demonstrate bone infiltration is a magnetic resonance. However, in some instances, the distinction between bone marrow edema and tumor infiltration could be difficult. In a recent article, it was published that out of 43 cases of sSCC treated with Mohs surgery, 12 underwent distal phalanx disarticulation because of voluminous tumor and radiological changes suggestive of bone invasion. From these 12 patients, only 6 cases were reported to have bone invasion after histopathological study of the amputated bone tissue [6]. In our cases, we documented histologically invasive sSSC, 1 associated with high-risk HPV infection.
In both cases, MRI reported doubtful features, impossible to differentiate between edema or bone infiltration by the tumor. When Mohs surgery is performed, periosteum can be examined during surgery, but in conventional surgery, instead of amputation, we decided to perform a bone biopsy during the wide surgical excision of the nail unit, planning that if the histopathological study was positive for bone infiltration, a second surgery should be performed to amputate the distal phalanx. If there were no signs of bone involvement, the digit could be preserved preventing high morbidity secondary to this type of surgical process. In both cases, the bone was free of tumor infiltration, and both patients conserved their digit phalanx. After 18 months of follow-up, both of them are free of disease.

\section{Conclusion}

In this paper, it is proposed that in the presence of invasive sSCC with radiological image studies of uncertain osseous infiltration, a bone biopsy could be performed during the en bloc excision of the nail unit. Then, after histopathological confirmation of tumor invasion of the bone, phalanx amputation surgery is mandatory.

\section{Statement of Ethics}

Both patients gave their written informed consent to publish their case and photos. Also, the research was conducted ethically in accordance with the World Medical Association Declaration of Helsinki.

\section{Conflict of Interest Statement}

The author has no conflicts of interest to declare.

\section{Funding Sources}

There were no funding sources for the preparation of this manuscript.

\section{Author Contributions}

Dr. Dominguez-Cherit: substantial contributions to the conception or design of the work; acquisition, analysis, or interpretation of data for the work; contributions to the surgery and followup of the patients. The author agrees to be accountable for all aspects of the work. 


\section{References}

1 Topin-Ruiz S, Surinach C, Dalle S, Duru G, Balme B, Thomas L. Surgical treatment of subungual squamous cell carcinoma by wide excision of the nail unit and skin graft reconstruction: an evaluation of treatment efficiency and outcomes. JAMA Dermatol. 2017; 153(5):442-8.

2 Dijksterhuis A, Friedeman E, van der Heijden B. Squamous cell carcinoma of the nail unit: review of the literature. J Hand Surg Am. 2018;43(4):374-e2.
3 Starace M, Alessandrini A, Dika E, Piraccini BM. Squamous cell carcinoma of the nail unit. Dermatol Pract Concept. 2018 Jul;8(3):23844.

4 National Comprehensive Cancer Network (2020) Clinical Practice Guidelines in Oncology: Non-Melanoma Skin Cancer: Squamous Cell Skin Cancer Version 1.2020 Retreived from https://www.nccn.org/professionals/ physician_gls/pdf/squamous.pdf.
5 Cañueto J, Burguillo J, Moyano-Bueno D, Viñolas-Cuadros A, Conde-Ferreirós A, Corchete-Sánchez LA, et al. Comparing the eighth and the seventh editions of the American Joint Committee on Cancer staging system and the Brigham and Women's Hospital alternative staging system for cutaneous squamous cell carcinoma: implications for clinical practice. JAAD. 2019;80(1):106-13.e2.

6 Dika E, Fanti PA, Ptrizi A, Misciali C, Vaccari S. Piraccini BM Mohs surgery for squamous cell carcinoma of the nail unit: 10 years of experience. Derm Surg. 2015;41(9):1015-9. 